\title{
A Case of Giant Aortoiliac Aneurysm Rupture when Open Repair Seems a One-Way Street
}

\author{
${ }^{1}$ Department of Vascular Surgery, Metropolitan General Hospital, \\ Athens, Greece \\ ${ }^{2}$ Department of Vascular Surgery, Athens Medical Center, Athens, \\ Greece \\ ${ }^{3}$ Division of Vascular Surgery, Sismanoglio-Fleming Hospital of \\ Athens, Athens, Greece
}

Georgios Galanopoulos, MD, PhD, MSc ${ }^{1,2}$ Vassilios Papavassiliou, MD, PhD 3

\begin{abstract}
Address for correspondence Georgios Galanopoulos, MD, PhD, MSc, 21, Venizelou Street, 15127, Melissia, Athens 15127, Greece (e-mail: georgiosgalanopoulos@yahoo.com).
\end{abstract}

AORTA 2020;8:172-174.
Abstract
Keywords
- aortoiliac
- aneurysm
- rupture
- giant
- open
- repair

Giant aortoiliac aneurysm is a rare nosological entity. Owing to the increased diameter, the risk of rupture is extremely high and, similarly, the repair is extremely challenging. In this article, open surgical repair of a ruptured giant aortoiliac aneurysm in a 72-yearold male is described. A bifurcated Dacron graft was used with left internal iliac artery revascularization, while the contralateral internal iliac artery was ligated. The patient had an uneventful recovery.

\section{Introduction}

The natural history of an aortoiliac aneurysm (AIA), as with every aneurysm, is progressive expansion and at the end, if not treated, rupture. Rupture is a catastrophic complication because it incurs an extremely high mortality rate. Most patients with ruptured AIA do not reach the hospital. Those who survive to be subjected to an intervention have an elevated mortality and morbidity rate. The annual risk of rupture increases with aneurysm expansion. It is calculated that the annual risk of rupture of an abdominal aortic aneurysm (AAA) with a diameter of $>70 \mathrm{~mm}$ is 30 to 33\%. ${ }^{1}$ Ruptured AIA represents a special surgical challenge. Specific surgical skills are required. Giant AIAs are defined as those having a transverse diameter $>13 \mathrm{~cm}$. Herein, a case of a giant ruptured AIA treated successfully is described.

\section{Case Presentation}

A 72-year-old man presented to the emergency department with pain in his right lower abdominal quadrant of 4 hours. His past

received

August 7, 2019

accepted after revision

September 30, 2020

published online

March 24, 2021 medical history was significant for smoking (50 packs/year) and poorly controlled hypertension. He had no history of previous abdominal surgery. On admission, the patient had hypotension $(84 / 46 \mathrm{~mm} \mathrm{Hg}$ ) and tachycardia (106 beats/min). Palpation of his abdomen revealed a large, tender pulsatile mass around the umbilicus.

Emergency contrast-enhanced computed tomography revealed a giant infrarenal AAA extending to both common iliac arteries ( - Fig. 1 ) with evident rupture at the medial wall of the right iliac aneurysm (-Fig. 2). This AIA measured $17 \mathrm{~cm} \times 10 \mathrm{~cm}$ at the aortic bifurcation, $12 \mathrm{~cm} \times 10 \mathrm{~cm}$ at the ruptured common right iliac artery aneurysm, and $9 \mathrm{~cm} \times 8 \mathrm{~cm}$ at the left common iliac aneurysm. The aortic and both iliac aneurysms presented with extensive thrombus within the aneurysmal sacs. Both internal iliac arteries were patent.

The patient was immediately transferred to the operating theater.

An open transperitoneal approach was chosen. Through a midline incision, aortic cross-clamping was performed within a few minutes. Both external iliac arteries were dissected and controlled. Patent internal iliac arteries were controlled from within with inflatable balloons after opening the AIA. A

\section{(c) 2021. The Author(s).}

This is an open access article published by Thieme under the terms of the Creative Commons Attribution License, permitting unrestricted use, distribution, and reproduction so long as the original work is properly cited. (https://creativecommons.org/licenses/by/4.0/)

Thieme Medical Publishers, Inc., 333 Seventh Avenue, 18th Floor, New York, NY 10001, USA 


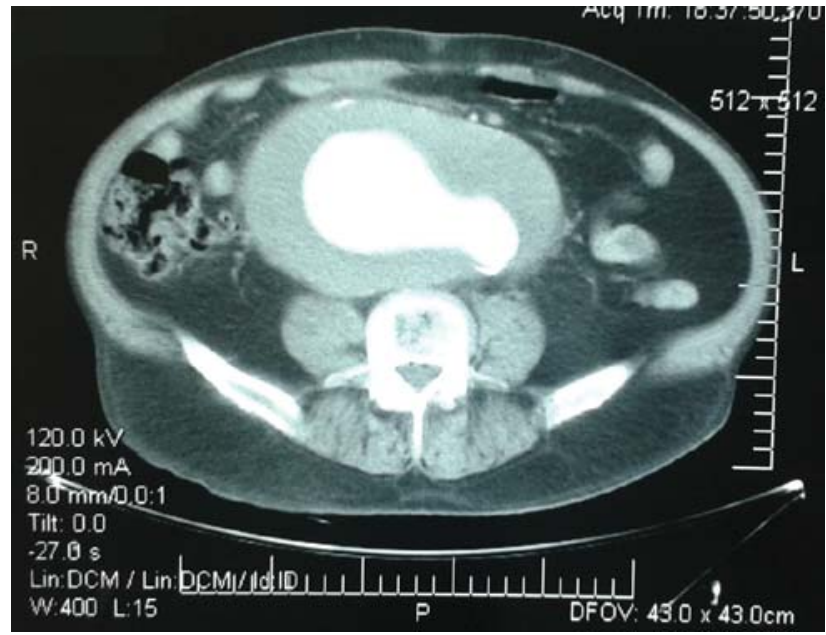

Fig. 1 Contrast-enhanced computed tomography demonstrates a giant aortoiliac aneurysm at the level of the aortic bifurcation with extensive thrombus.

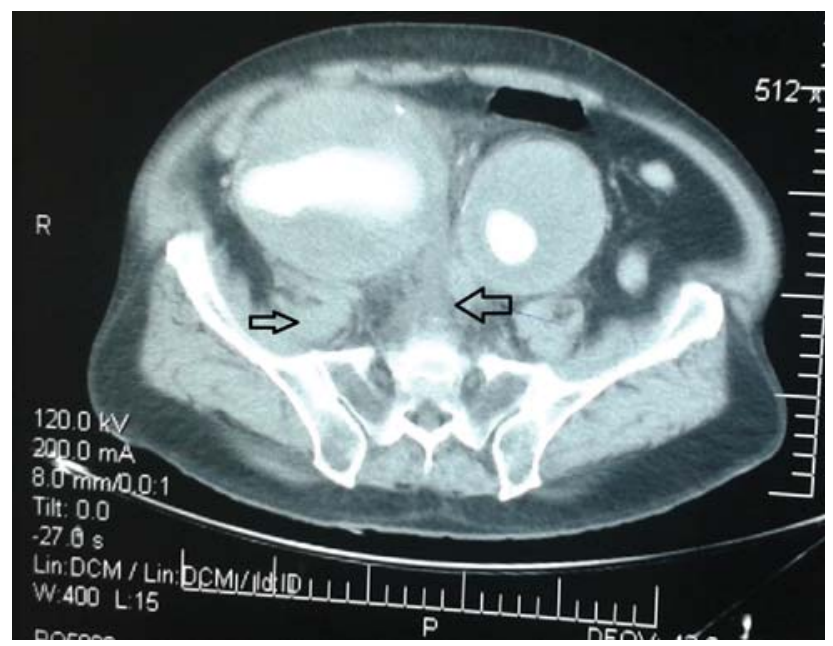

Fig. 2 Appearance of giant iliac aneurysms with extensive thrombus and signs of rupture in the medial wall of the right iliac artery. Significant retroperitoneal pelvic hematoma is shown (arrows).

Y-shaped Dacron graft $18 \mathrm{~mm} \times 9 \mathrm{~mm}$ was used. Proximal anastomosis at the infrarenal aorta was performed just below the renal arteries. Distal anastomoses were performed in an end-to-end fashion with the external iliac arteries. The right internal iliac artery was oversewn from within at the ostium, while the left was revascularized via a jump graft from the iliac limb of the bifurcated graft. The jump graft was anastomosed in an end-to-end fashion to the ostium of the left internal iliac artery.

At the end of the procedure, the sigmoid colon had no signs of ischemia. During surgery, the patient was stable and received 8 units of packed red blood cells.

His postoperative course was uneventful and he was discharged from hospital on postoperative day 8 . The patient continues to do well 3 years later. The last follow-up ultrasound imaging at 3 years from surgery revealed normal findings (-Figs. 3 and $\mathbf{4}$ ).

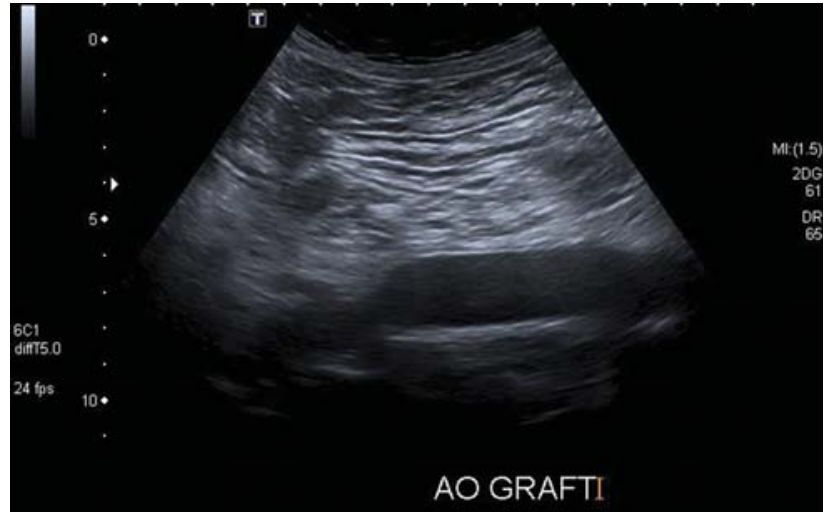

Fig. 3 B-mode image of the proximal anastomosis.

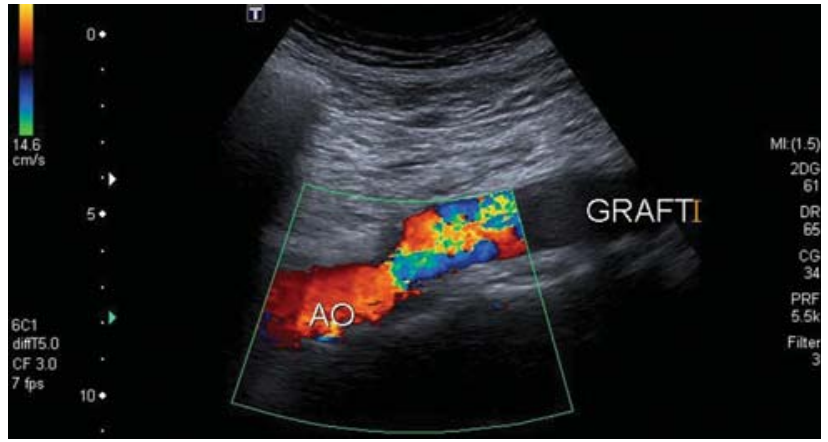

Fig. 4 Color-flow Doppler image of the proximal anastomosis.

\section{Discussion}

In the literature, giant AAAs occur almost exclusively in male patients. Clear predilection for male sex also characterizes aortic and iliac aneurysmal disease. The incidence of ruptured AIA seems to have declined in recent years, and this is partly due to the proliferation of screening in the population. The prevalence of giant AIAs is hard to estimate and is largely unknown. There are only a few reports in the literature, which means that giant AIAs are infrequently detected. The aforementioned screening protocols aim to timely detect AAAs in the population, recommending repair or surveillance according to their diameter, thus limiting the possibility for an aneurysm to excessively expand.

Although rupture rates are high for AAAs measuring $>7 \mathrm{~cm}$, strangely enough a small number of them, due to unidentified protective factors, do not rupture and continue to grow reaching extreme diameters.

The diameter and shape of the aneurysm are determining factors, closely related to the risk of rupture.

Sandhu and Pipinos ${ }^{2}$ categorized iliac artery aneurysms based on their anatomical features. Combined aneurysms of the common iliac arteries and abdominal aorta are categorized as Type-E aneurysms and may be treated with bifurcated grafts.

The decision to take when facing with ruptured AIA concerns whether to treat it by open or endovascular means. Open repair is still the gold standard and always bears consideration in ruptured aneurysms. In experienced hands, complication rates are significantly reduced. Obviously, the 
presence of a giant AIA notably obfuscates the surgical field, making surgical maneuvers more demanding. Adjacent organs become adherent due to the extreme dimensions of the aneurysm, making dissection more difficult. On the other hand, endovascular repair has several limitations related to the anatomy of the diseased vessels, especially of the infrarenal aortic neck. Extreme angulation, large diameter, short length, and significant thrombus burden, all may accompany the extreme dimensions of the aneurysmal sac. ${ }^{3}$ The anatomical suitability for endovascular treatment of ruptured AAA is reported at $46 \%{ }^{4}$ Additionally, in the EUROSTAR (European collaborators on stent/graft techniques for aortic aneurysm repair) registry, larger (nonruptured) aneurysms were associated with increased incidence of endoleaks following endovascular repair, especially when there is concomitant aneurysmal pathology in abdominal aorta and iliac arteries. $^{5}$ In the IMPROVE trial (immediate management of patients with rupture: open versus endovascular repair), the 30-day mortality is similar among patients treated by open or endovascular means, with a shorter length of stay for those treated endovascularly. ${ }^{6}$

The decision on how to treat a patient presenting to the emergency department with ruptured AIA is quite complex and multifactorial. This depends mainly on the experience of the operating doctor and his team. Special technical expertise is required. For both approaches if the experience is larger in one of the two treatment modalities, this is the case not to try the other one. If the availability of stent grafts, wires, catheters, balloons is limited, the endovascular way should not be attempted. Also, that endovascular treatment in emergency cases requires a readily available multidisciplinary staff with dedicated equipment. If there is no time to size the aneurysms and choose the best endograft, open repair is the only way.

In our particular case of giant ruptured AIA, the choice to immediately transfer the patient in the operating theater and obtain rapid aortic control represented an undoubtedly salvage procedure.

We are not aware of other reports of a giant aortic aneurysm measuring as much as $17 \mathrm{~cm}$ in transverse diameter combined with giant right and left common iliac aneurysms of as large as 12 and $9 \mathrm{~cm}$, respectively.

The presence of bilateral common iliac artery aneurysms may be a limitation for endovascular treatment, due to the need for embolization of both internal iliac arteries to prevent retrograde flow and subsequently continuous bleeding. The risk of pelvic ischemia, with buttock claudication, bowel and urinary bladder ischemia, or erectile dysfunction, is high when both internal iliac arteries are sacrificed. On the other hand, the use of branched iliac devices combined with aortic bifurcated stent, grafts did not seem a safe option in our emergency situation. These procedures may be time consuming. Finally, it has been observed that concomitant aortic and common iliac aneurysms treated by endovascular means incur a high rate of endoleaks. ${ }^{5}$ For this reason, prophylactic secondary interventions are frequently needed.

Open repair of AAAs may be related with several complications that become more prominent when dealing with giant AAAs. Significant displacement of adjacent organs enhances the risk of iatrogenic injuries especially when rupture of such aneurysms occurs. Venous injuries or small bowel serosal tears may also occur. To avoid such unpleasant surprises, cautious dissection after aortic cross clamping is mandatory, taking care to identify meticulously the surrounding anatomic structures. Additionally, pelvic revascularization in the presence of giant internal iliac aneurysms may be extremely challenging due to the limited surgical field in association with their location deep in the pelvis. Attempts to preserve at least one internal iliac artery should be made, as in our case.

Funding

None.

Conflict of Interest

The authors declare no conflict of interest related to this article.

\section{Acknowledgments}

None.

\section{References}

1 Moll FL, Powell JT, Fraedrich G, et al; European Society for Vascular Surgery. Management of abdominal aortic aneurysms clinical practice guidelines of the European society for vascular surgery. Eur J Vasc Endovasc Surg 2011;41(Suppl 1):S1-S58

2 Sandhu RS, Pipinos II. Isolated iliac artery aneurysms. Semin Vasc Surg 2005;18(04):209-215

3 Cho J, Jung H, Kim HK, Huh S. Open repair of ruptured huge aorto iliac aneurysm: warning of colon ischemia. Vasc Spec Int 2014;30 (02):76-79

4 Hoornweg LL, Wisselink W, Vahl A, Balm R, Amsterdam Acute Aneurysm Trial Collaborators. The Amsterdam Acute Aneurysm Trial: suitability and application rate for endovascular repair of ruptured abdominal aortic aneurysms. Eur J Vasc Endovasc Surg 2007;33(06):679-683

5 Hobo R, Sybrandy JE, Harris PL, Buth J, EUROSTAR Collaborators. Endovascular repair of abdominal aortic aneurysms with concomitant common iliac artery aneurysm: outcome analysis of the EUROSTAR Experience. J Endovasc Ther 2008;15(01):12-22

6 Powell JT, Sweeting MJ, Thompson MM, et al; IMPROVE Trial Investigators. Endovascular or open repair strategy for ruptured abdominal aortic aneurysm: 30 day outcomes from IMPROVE randomised trial. BMJ 2014;348:f7661 